\title{
Evaluation of accuracy of plain radiography in determining the Risser stage and identification of common sources of errors
}

\author{
Jae Hyuk Yang ${ }^{1}$, Amit Wasudeo Bhandarkar' ${ }^{1}$ Seung Woo Suh'1, Jae Young Hong ${ }^{2}$, Jin Ho Hwang ${ }^{3 *}$ \\ and Chang Hwa Ham
}

\begin{abstract}
Background: Risser's sign is an established radiological marker for predicting growth potential in adolescent idiopathic scoliosis, but the accuracy of Risser's staging has been debated. This research was designed to evaluate the accuracy of Risser's staging and to identify causes of errors in Risser's staging.

Materials and methods: Plain radiographs of 89 adolescent idiopathic scoliosis patients were evaluated for Risser's stage using both the Original and French methods. A three-dimensional computed tomography (3D-CT) was used to evaluate the accuracy of the plain radiographs. Inter- and intra-observer reliability of both methods was assessed on radiographs and 3D-CT images using weighted kappa statistics. The concordance rate for Risser's staging between plain radiographs and 3D-CT images were calculated. The various sources of staging differences between the two imaging methods were noted, grouped, and analyzed to identify common error patterns.
\end{abstract}

Results: Intra- and inter-observer staging reliabilities on plain radiography were 0.91 and 0.94 , respectively, using the Original method and 0.91 and 0.92 , respectively, using the French method. Intra- and inter-observer reliabilities on 3D-CT were 0.98 and 0.99 , respectively, using the Original method and 0.97 and 0.99 , respectively, using the French method. Mean concordance rates between plain radiography and 3D-CT were $59.76 \%$ and $67.42 \%$ using the Original and French methods, respectively. Common sources of error leading to misinterpretation of Risser's staging were miscalculation of apophysis excursion, skip ossification, isolated non-linear ossification, micro-fusion, and pseudo-fusion.

Conclusions: Risser's staging by plain radiography is reliable but not accurate. Variations in the iliac apophysis ossification and misinterpretation of apophysis fusion are the main sources of error.

Keywords: Pelvis, Ossification, Risser's sign, Computed tomography, Concordance rate

\section{Introduction}

In 1936, Joseph C. Risser described the capping and progression of iliac apophysis ossification as an invaluable aid in determining spinal skeletal maturity [1]. As there is a strong correlation between vertebral column growth and the length of iliac apophyseal ossification, excursion, and fusion, Risser's sign became an established radiological marker for predicting curve progression in adolescent idiopathic scoliosis (AIS) patients [1]. Commonly, only an

\footnotetext{
*Correspondence: scoliosis@yuhs.ac

${ }^{3}$ Division of Pediatric Orthopaedics, Orthopaedic Surgery, Yonsei University

College of Medicine, Severance Children's Hospital, Seoul, Korea

Full list of author information is available at the end of the article
}

anteroposterior pelvic radiograph is assessed to calculate Risser's stage of an individual; however, the reliability and accuracy of Risser's staging by plain radiography $(P R)$ have been questioned [2]. Understanding that a strong correlation exists between skeletal growth and the sequence of iliac apophyseal ossification, we hypothesized that the common Risser staging method involving $P R$ is not accurate and often results in miscalculation of the iliac apophyseal excursion, ossification, and fusion due to superimposition of the iliac bone and apophysis. To overcome the assumed limitations, some authors have suggested the inclusion of additional views of the ilium for improved assessment of the iliac apophysis; however, 
despite these new studies, the reliability and accuracy of Risser's staging remain inconsistent and controversial [3-6]. Three-dimensional computed tomography (3D-CT), which overcomes all of the limitations of two-dimensional imaging, is undoubtedly the best tool to assess actual changes in the iliac apophysis and may be the best tool to determine the accuracy of Risser's staging [7-9]. We utilized 3D-CT to evaluate the accuracy of Risser's staging performed using $P R$ in a homogenous population of AIS patients. An added objective was to identify common patterns and causes of error in the interpretation of iliac apophysis excursion by $P R$ and to identify improvements to Risser's staging system using PR.

\section{Materials and methods}

After obtaining the approval from the Institutional Review Board, a retrospective review of patients who underwent corrective surgery for idiopathic scoliosis between 2004 and 2009 was performed. As a routine scoliosis assessment, these patients subjected to a series of standard whole spine radiographs. As part of our standard institutional protocol, whole spine radiographs were acquired on a long cassette with the patient in an erect position so that the iliac crest apophysis could be visualized completely. The 3D-CT was not performed routinely but was offered to the patients as an option for improved preoperative planning. Between 2004 and 2009, 3D-CT scanning was used for preoperative planning in the 89 patients that were included in our study. Before 3D-CT evaluation, all patients were informed of the risk of radiation exposure, and all patients provided informed consent.

A 3D-CT evaluation was performed with a 16-channel instrument (Somatom Sensation 16, Siemens AG, Erlangen, Germany) using the following protocol: $120 \mathrm{kV}, 80 \mathrm{mAs}$, $5.63 \mathrm{CTDI}_{\mathrm{vol}}$, 432 DLP, and CAREDOSE modulation. Axial cuts were acquired at $2 \mathrm{~mm}$ intervals from the cervical spine to the pelvis and were used in reconstruction of the coronal, sagittal, and three-dimensional views of the whole spine and pelvis [8]. Coronal sections reconstructed at $2 \mathrm{~mm}$ intervals were also used to evaluate the shape of the deformity, size of the pedicle, and the fusion of apophysis in greater detail [8].

\section{Risser's staging}

Two fellowship-trained orthopedic spinal surgeons evaluated Risser's staging on $P R$ and 3D-CT scans using the Original method $(O M)$ and the French method (FM) [10]. In the $O M$ of Risser's staging, which is practiced in the United States, the iliac wing is divided into the following six grades: Risser 0, no ossification; Risser 1, ossification within the first quarter of the crest (up to 25\%); Risser 2, ossification extending into the second quarter of the crest $(25 \%-50 \%)$; Risser 3, ossification into the third quarter of the crest (50\%-75\%); Risser 4, ossification into the fourth quarter of the crest (more than $75 \%$ ) to completion of the apophyseal line excursion; and Risser 5, fusion of the apophyseal ring to the ilium, from the start of the process posterior-medially to its completion [10].

In the $F M$ of Risser's staging, which is widely used in Europe, the iliac crest is divided into three parts, and there are six grades of staging as follows: Risser 0, no ossification; Risser 1, ossification within the first anterior third (33\%); Risser 2, ossification extending into the second third (33\%-66\%); Risser 3, ossification of the entire apophysis $(66 \%-100 \%)$; Risser 4 , beginning of fusion of the apophysis to the ilium posterior-medially; and Risser 5 , complete fusion of the apophysis to the ilium [10].

The Risser staging was performed on a whole spine anterior-posterior AP view using the two traditional methods. While performing the Risser staging using a 3D-CT scan, the widest view of the iliac bone was obtained by rotating the 3D images to evaluate the true excursion ratio between the apophysis and the iliac crest. The iliac crest was divided into four equal sections in the $O M$ and into three equal sections in the $F M$. The length of the iliac apophysis ossification was described as a percentage of the total length of the iliac crest. If there was a doubt regarding fusion of the apophysis, it was confirmed on coronal sections.

Additionally, while evaluating ossification of the iliac apophysis on 3D-CT, certain variations in patterns of ossification, excursion, and fusion of the iliac apophysis were observed and were classified as conventional ossification, skip ossification, isolated non-linear ossification, micro-fusion, or pseudo-fusion. Conventional ossification was defined as ossification of the iliac crest progressing in a linear pattern from anterior to posterior, while skip ossification was defined as a step (discontinuity) in the ossification of the iliac apophysis. Isolated non-linear ossification was described as only round-shaped, nonlinear ossification in the anterior or posterior aspect of the iliac crest, and micro-fusion was defined as- anterolateral or posteromedial fusion visualized on coronal cut or 3D reconstructed $\mathrm{CT}$ images, with no fusion seen on plain radiography regardless of apophyseal excursion. Lastly, pseudo-fusion was defined as the presence of a clear sclerotic line at the posterior iliac spine on $P R$ but absence of fusion on coronal cuts or $3 \mathrm{D}$ reconstructed images of CT.

Two orthopedic surgeons who were blinded to the measurements repeated Risser's staging three times using $\mathrm{OM}$ and FM on $P R$ and 3D-CT images at oneweek intervals. If there was disagreement over the staging of Risser's sign, the final Risser stage was determined after a discussion but before the calculation of concordance rates. The data was then reevaluated by an 
Table 1 Analysis of concordance between radiography and computed tomography according to Risser's stage using the Original method

\begin{tabular}{|c|c|c|c|c|c|c|c|}
\hline \multirow{2}{*}{$\begin{array}{l}\text { Stage determined by CT } \\
\text { Stage }\end{array}$} & \multicolumn{7}{|c|}{ Stage determined by plain radiography } \\
\hline & 0 & 1 & 2 & 3 & 4 & 5 & Total \\
\hline 0 & 11 & 1 & 0 & 0 & 0 & 0 & 12 \\
\hline 1 & 3 & 0 & 2 & 0 & 0 & 0 & 5 \\
\hline 2 & 0 & 2 & 5 & 0 & 0 & 1 & 8 \\
\hline 3 & 0 & 0 & 2 & 1 & 1 & 1 & 5 \\
\hline 4 & 0 & 0 & 1 & 5 & 15 & 14 & 35 \\
\hline 5 & 0 & 0 & 0 & 0 & 3 & 21 & 24 \\
\hline Total & 14 & 3 & 10 & 6 & 19 & 37 & 89 \\
\hline Concordance rate & $78.57 \%$ & $0.00 \%$ & $50.00 \%$ & $16.67 \%$ & $78.95 \%$ & $56.76 \%$ & $59.55 \%$ \\
\hline
\end{tabular}

The distribution of error types was as follows: miscalculation of excursion $(n=12)$, skip ossification $(n=3)$, isolated non-linear ossification ( $n=3$ ), micro-fusion $(n=3)$, pseudo-fusion $(n=14)$, and presence of gas shadow $(n=1)$. Further, the results of the statistical analyses revealed a significant difference between stages $(p=0.011)$, with stages $1,2,3$, and 5 exhibiting a lower rate of concordance $(<70 \%)$. According to $F M$, concordance rates were $78.57 \%$ in stage $0,40.00 \%$ in stage $1,66.67 \%$ in stage $2,80.95 \%$ in stage $3,36.00 \%$ in stage 4 , and $100 \%$ in stage 5 (Table 2 ).

CT computed tomography, \% percentage.

independent radiologist who did not participate in the study. Thereafter, the concordance rate of Risser's staging between $P R$ and 3D-CT was calculated.

\section{Statistical evaluation}

Weighted kappa statistics were used to analyze 3D-CT and $P R$ intra- and inter-observer Risser's staging reliability values and results were expressed as kappa $(\mathrm{\kappa})$ values. A kappa value greater than 0.81 was considered nearly perfect agreement, $0.61-0.80$ as substantial agreement, $0.6-0.41$ as moderate agreement, $0.4-0.21$ as fair agreement, $0.2-0.01$ as slight agreement, and a value below 0.01 as poor agreement [11].

The concordance rate of each Risser's stage was evaluated using Fisher's exact test. A $p$ value less than 0.05 was considered statistically significant. SPSS 13.0 for Windows (SPSS Inc., IL, USA) was used for the statistical analysis.

\section{Results}

The study included 27 males and 62 females. The mean chronological age was $12.3 \pm 5.9$ years (range, 10-18 years). The distribution of enrolled patients according to the method of Risser's staging is described in Tables 1 and 2. All Risser's stage PR and 3D-CT intra- and interobserver reliability values were greater than 0.9 regardless of the measurement method (Table 3).

According to the results using $O M$, concordance rates were $78.57 \%$ in stage $0,0.0 \%$ in stage $1,50.00 \%$ in stage $2,16.67 \%$ in stage $3,78.95 \%$ in stage 4 , and $59.55 \%$ in stage 5 (Table 1).

\section{Discussion}

The Risser staging system is widely used to assess the potential for progression of spinal curvature in AIS in combination with other predictive factors such as triradiate cartilage closure, mean height velocity, Tanner's

Table 2 Analysis of concordance between radiograph and computed tomography according to Risser's stage determined using the French method

\begin{tabular}{|c|c|c|c|c|c|c|c|}
\hline Stage determined by CT & Stage $d$ & ed by $\mathrm{pl}$ & ography & & & & \\
\hline Stage & 0 & 1 & 2 & 3 & 4 & 5 & Total \\
\hline 0 & 11 & 1 & 0 & 0 & 0 & 0 & 12 \\
\hline 1 & 3 & 2 & 1 & 0 & 0 & 0 & 6 \\
\hline 2 & 0 & 1 & 6 & 0 & 2 & 0 & 9 \\
\hline 3 & 0 & 1 & 2 & 17 & 10 & 0 & 30 \\
\hline 4 & 0 & 0 & 0 & 4 & 9 & 0 & 13 \\
\hline 5 & 0 & 0 & 0 & 0 & 4 & 15 & 19 \\
\hline Total & 14 & 5 & 9 & 21 & 25 & 15 & 89 \\
\hline Concordance rate & $78.57 \%$ & $40.00 \%$ & $66.67 \%$ & $80.95 \%$ & $36.00 \%$ & $100.00 \%$ & $67.42 \%$ \\
\hline
\end{tabular}

The distribution of different types of error using FM was as follows: miscalculation of excursion ( $n=4)$, skip ossification ( $n=3$ ), isolated non-linear ossification $(n=2)$, micro-fusion $(n=8)$, pseudo-fusion $(n=10)$, and gas shadow $(n=2)$. The results of the statistical analyses revealed a significant difference between stages $(p=0.0001)$, with stages 1,2 , and 4 exhibiting a lower rate of concordance $(<70 \%)$. The mean concordance rate between $P R$ and $3 D-C T$ for assessing Risser's staging was $59.76 \%$ for $O M$ and $67.42 \%$ for $F M$.

CT computed tomography, \% percentage. 
Table 3 Reliability of the Original and French methods for Risser's staging using $\mathrm{CT}$ or plain radiography

\begin{tabular}{lll}
\hline & $\begin{array}{l}\text { Inter-observer } \\
\text { reliability }\end{array}$ & $\begin{array}{l}\text { Intra-observer } \\
\text { reliability }\end{array}$ \\
\hline $\begin{array}{l}\text { Original method } \\
\text { (radiograph) }\end{array}$ & $0.94^{\mathrm{a}}$ & $0.91^{\mathrm{a}}$ \\
$\begin{array}{l}\text { Original method (CT) } \\
\begin{array}{l}\text { French method } \\
\text { (radiograph) }\end{array}\end{array}$ & $0.99^{\mathrm{a}}$ & $0.98^{\mathrm{a}}$ \\
$\begin{array}{l}\text { French method (CT) } \\
\text { Comparison of concordance rate between radiograph and CT }\end{array}$ & $0.91^{\mathrm{a}}$ \\
$\begin{array}{l}\text { according to Risser's stages } \\
\text { Original method }\end{array}$ & $0.000009^{\mathrm{b}}$ & \\
French method & $0.10^{\mathrm{b}}$ & \\
\hline
\end{tabular}

$A P$ anterior-posterior, $C T$ computed tomography.

${ }^{a}$ Weighted kappa statistics were used, and all values are expressed as kappa values.

${ }^{\mathrm{b}}$ Fisher's exact test was used, and all values are expressed as $p$ values.

staging, radiographs of hand and elbow, and chronological age [12]. Risser's staging is the most commonly used method for assessing skeletal maturity because it is easily determined using pelvic radiography. Likewise, Risser's staging is a well-established follow-up tool for determining the acceleration and cessation of vertebral growth in AIS patients and is widely used for research purposes.

However, several reports in the literature have suggested that the Risser staging system is less accurate for predicting vertebral growth than hand-wrist radiography, skeletal age, height, and even chronological age $[6,7,12,13]$. There are also doubts regarding the reliability and accuracy of Risser's staging when assessed using only posteroanterior plain radiographs, because ossification of the iliac apophysis is best viewed on anteroposterior radiographs. Izumi et al. reported only $58 \%$ agreement between posteroanterior and anteroposterior Risser's staging [3]. A hypothesis in the current study was that one of the reasons for the low accuracy of Risser's staging in the determination of spinal growth potential might be inaccurate identification of the Risser stage itself. In the literature, inaccurate staging has been attributed to inability to completely visualize the iliac apophysis due to superimposition of the iliac bone in frontal radiographs and to variations in iliac apophysis excursions, fusions, and ossifications [3-6]. Reports on the frequency of such variations range from $10 \%$ to $41 \%$. Risser observed fragmentary development of the iliac apophysis with interruptions that later resolved the appearance of iliac epiphysis at the posterior side (1\%) and variable excursions of the apophysis in $10 \%$ of cases. Zaoussis and James observed apophysis with short excursions in $10 \%$ of cases, early fusion of the apophysis in $24 \%$, and posterior ossification in $40 \%$ of cases. In their study of 34 patients, Shuren et al. found five reverse progressions, five cases of capping in fragments, including three cases that started capping toward the middle, and one case of partial fusion during capping. Such anomalous patterns of iliac apophyseal ossification may introduce errors into Risser stage calculations and further decrease the accuracy of $P R$ for its calculation.

For the above reasons, some authors have suggested the use of lateral spinal radiographs in addition to plain pelvic radiographs to reliably detect iliac apophysis excursions and fusions and demonstrated that the added radiographs improved accuracy [5]. Other authors tried to increase the reliability of Risser's staging using anteroposterior and posteroanterior bending views but found no significant difference from conventional AP radiographs [6]. However, these studies had inherent limitations in detecting the apophysis ossification and fusion, because evaluations were performed using only $P R$, which is a two-dimensional modality [3-6]. In order to verify the accuracy of $P R$ for estimating the Risser's stage, it is necessary to compare the results of PR with those of other diagnostic tools that have high sensitivity and specificity in detecting ossification and fusion. For this reason, 3D-CT was selected for the current study because the reconstructed images allow accurate visualization of the excursions and fusion of the iliac apophysis. The 3D-CT also has increased sensitivity for detecting different variations in the usual pattern of iliac apophyseal ossifications, fusions, and excursions [7-9].

Variable Risser's staging inter- and intra-observer reliability values following application of either $O M$ or $F M$ have been reported in the literature. Generally, previous studies have indicated good inter- and intra-observer reliability and reproducibility for calculating Risser's stages

Table 4 Stage wise distribution of causes of misinterpretations of Risser's stage

\begin{tabular}{lll}
\hline Risser's stage & Original method & French method \\
\hline Stage 0 & Micro-ossification $(2)^{1}$ Gas shadow $(1)^{2}$ & Micro-ossification $(2)^{1}$ Gas shadow $(1)^{2}$ \\
Stage 1 & Skip ossification $(2)^{3}$ Gas shadow $(1)^{2}$ & Gas shadow $(1)^{2}$ Skip ossification $(2)^{3}$ \\
Stage 2 & Skip ossification $(1)^{3}$ Miscalculation of excursion $(4)^{4}$ & Skip ossification $(1)^{3}$ Miscalculation of excursion $(2)^{4}$ \\
Stage 3 & Miscalculation of excursion $(5)^{4}$ & Micro-fusion $(4)^{5}$ \\
Stage 4 & Miscalculation of excursion $(1)^{4}$ Micro-fusion $(3)^{5}$ & Skip ossification $(2)^{3}$ Pseudo-fusion $(10)^{6}$ Micro-fusion $(4)^{5}$ \\
Stage 5 & Skip ossification $(2)^{3}$ Pseudo-fusion $(14)^{6}$ & \\
\hline
\end{tabular}

The numbers inserted in superscripts stand for the figure numbers in which the entities were illustrated. 


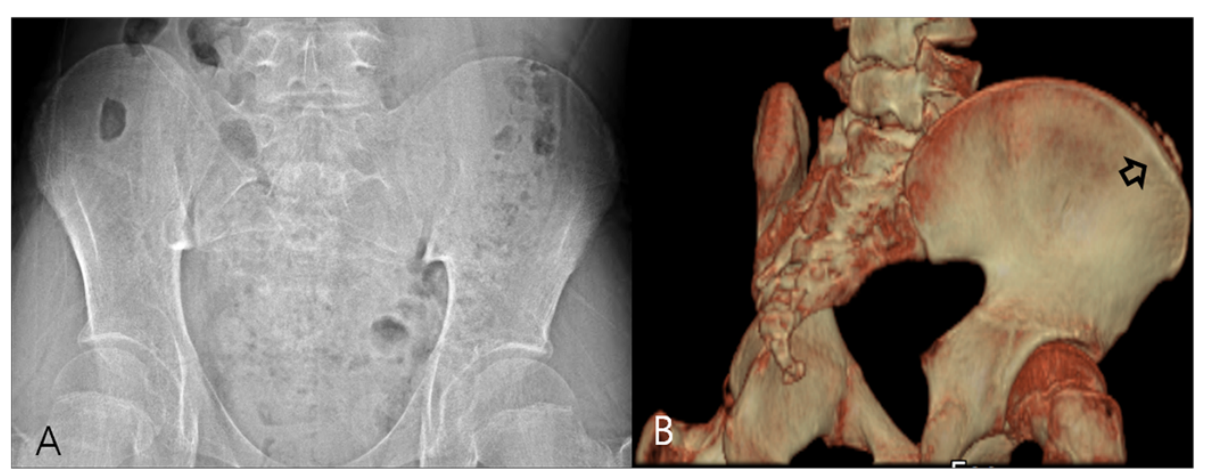

Figure 1 Micro-ossification. (A) Plain radiography performed on a 12-year-old boy did not reveal excursion of the apophysis and was graded as Risser stage 0. (B) A 3D-CT image of the same patient revealed micro-ossification of the iliac apophysis and was graded as Risser stage 1. The lower resolution and sensitivity of PR failed to show isolated non-linear ossification.

$[14,15]$. Dhar et al. [16] showed an inter-observer agreement of $89.2 \%$ and an intra-observer agreement of 93.4\%, while Goldberg et al. [2] obtained a kappa value of 0.80, which indicated excellent inter-observer agreement. Conversely, studies by Hammond et al. [17] and Shuren et al. [6] revealed only moderate agreement between radiologists and orthopedic surgeons for interpretation of Risser's stage and reported poor inter-observer reliability (kappa values of 0.31 and 0.53 , respectively).

The current study demonstrated high intra- and interobserver reliability between PR and 3D-CT for Risser's staging using both $O M$ and FM. However, the mean concordance rate between radiography and $3 \mathrm{D}-\mathrm{CT}$ was only $59.55 \%$ using $O M$ and $67.42 \%$ using FM (Table 3 ).

The results suggest that $P R$ was less accurate for Risser's sign staging when verified with $3 \mathrm{D}-\mathrm{CT}$. Furthermore, the concordance rates of Risser stages 1, 2, 3, and 5 in $O M$ and stages 1,2 , and 4 in $F M$ were less than $70 \%$.
Previous studies employed serial $P R$ to evaluate iliac apophyseal variations. Knowledge and awareness of these variations minimizes errors while staging. Serial CT evaluations of patients are not recommended, however, and the value of a single 3D-CT study when evaluating the exact nature of iliac apophyseal variations and their frequency is limited. With this in mind, patients with Risser's staging values from PR and CT that did not match were further analyzed to identify the reasons and sources of the errors. The following common sources of errors that might lead to misinterpretation in Risser's staging were identified: miscalculation of excursion, isolated non-linear ossification, skip ossification, pseudofusion, and micro-fusion. The distribution of errors that occurred at each stage while calculating Risser's grade using OM and FM is presented below. Risser's stage, as determined by a CT scan, was considered the real stage of the patient.

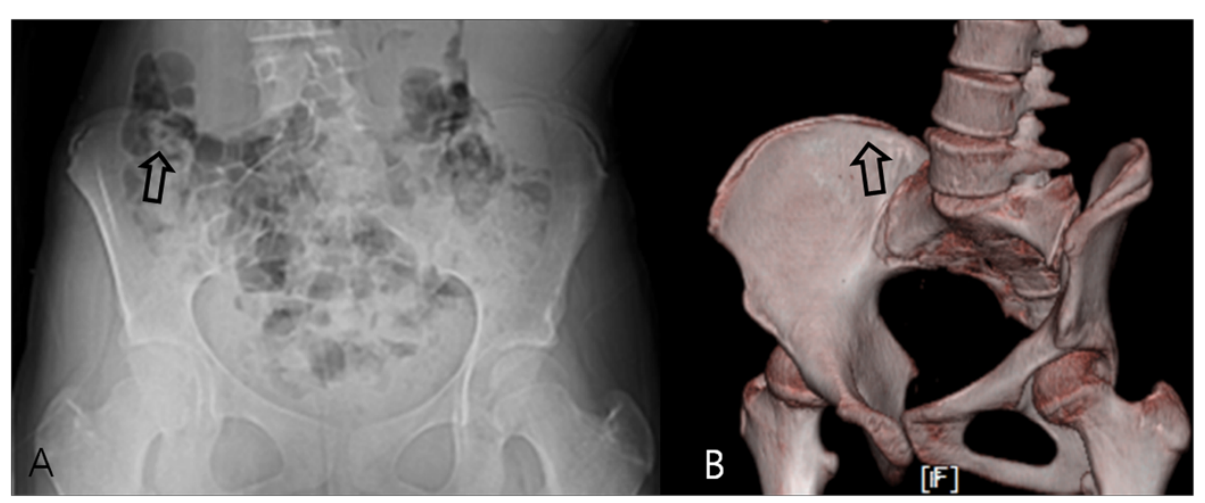

Figure 2 Gas shadow. (A) Plain radiography performed on a 13-year-old girl, a gas shadow on the posterior iliac crest prevented interpretation of bilateral excursion of the apophysis, and the patient was graded as Original Risser stage 2 and French Risser stage 1. (B) A 3D-CT image of the same patient, excursion of the iliac apophysis was clearly visible along the entire iliac bone, and was graded as Original Risser stage 4 and French Risser stage 3. Gas and internal organ shadows interfered with accurate staging. 


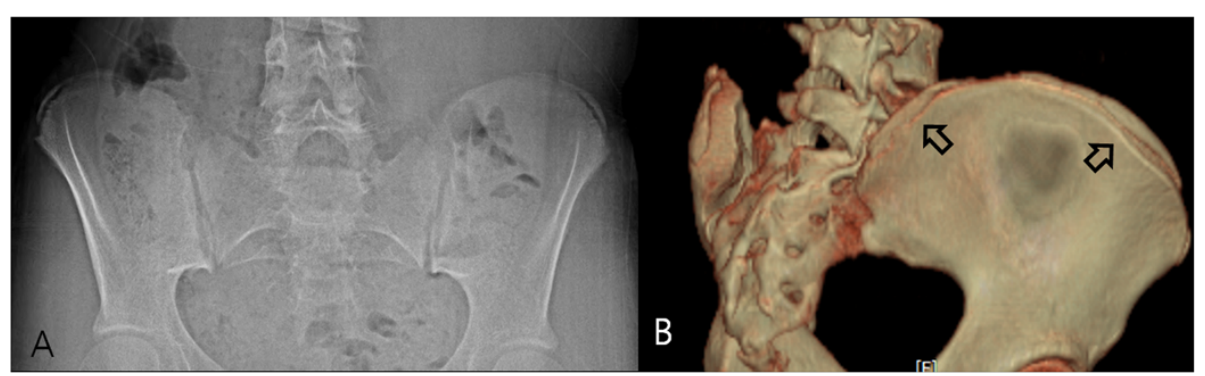

Figure 3 Skip ossification. (A) Plain radiography performed on a 14-year-old girl revealed excursion of the apophysis, approximately covering $60 \%$ of the iliac bone, and was graded as Original Risser stage 3 and French Risser stage 2. (B) A 3D-CT image of the same patient. Showing skipped ossification at the posterior iliac crest with grading of Original Risser stage 4 and French Risser stage 3. Initial apophyseal ossification of the ilium was not linear, but occurred in a skip pattern, which was not visualized properly by plain radiography.

Risser stage 0 on PR by both methods was determined to be Risser stage 1 on a CT scan due to micro-ossifications and gas shadows. The inaccuracy of Risser stage 1 on PR was graded differently on 3D-CT. The main cause for the discrepancy was the presence of skip ossification of the iliac apophysis, which led to an underestimation of the Risser's stage. Additionally, one patient had a soft organ shadow that was interpreted as ossification (Table 4, Figures 1, 2, 3).

For Risser stage 3 determined using PR, the main cause of error was different in OM and FM, which reflected the difference in the two staging systems. In $O M$, stage 3 is the anterior to posterior changing point for the excursion direction, whereas in $F M$, stage 3 is defined as full excursion of the apophysis without fusion. Hence, the most common cause of error in OM was miscalculation of excursion (Figure 4), whereas difficulty detecting micro-fusion was the most frequent source of error in FM. In Risser stages 4 and 5 determined using PR, the main cause of error was again different for $\mathrm{OM}$ and FM and was due to disparities in the staging systems. For OM, the main cause of error resulted from the inability to detect micro-fusion in stage 4 and pseudofusion in stage 5 (Table 4). However, the main cause of FM stage 4 errors was pseudo-fusion, whereas stage 5 exhibited perfect concordance between radiography and 3D-CT. In many cases, despite the observation of only a lucent posteromedial physeal line on PR, 3D-CT clearly demonstrated micro-fusion (Figure 5A,B). Meanwhile, in cases of a clear posteromedial sclerotic line, which suggested fusion on radiographs, 3D-CT demonstrated a clear lack of fusion on coronal cuts (i.e., pseudo-fusion; Figure 6A,B). Errors in both micro-fusion and pseudofusion occurred because PR lacks the level of sensitivity required for the accurate detection of iliac apophysis ossification and fusion. In addition, pelvic rotation, which is present in mild to moderate scoliosis deformities, adds to the spatial distortion. When analyzing $O M$ and FM concordance rates according to their stage, the concordance rates of $F M$ were more constant than the resulting $O M$ rates. A possible explanation for the divergent concordance rates might be the difference in the division of the iliac apophysis employed by each of the two methods (division into four portions in $O M$ and into three portions in $F M$ ). Errors caused by low resolution and the subsequent reduced capacity of PR to detect fusions, including pseudo-fusions and micro-fusions, were greater in stage 5 using $\mathrm{OM}$ and in stage 4 using FM. These stages signify the early fusion period during which fusion

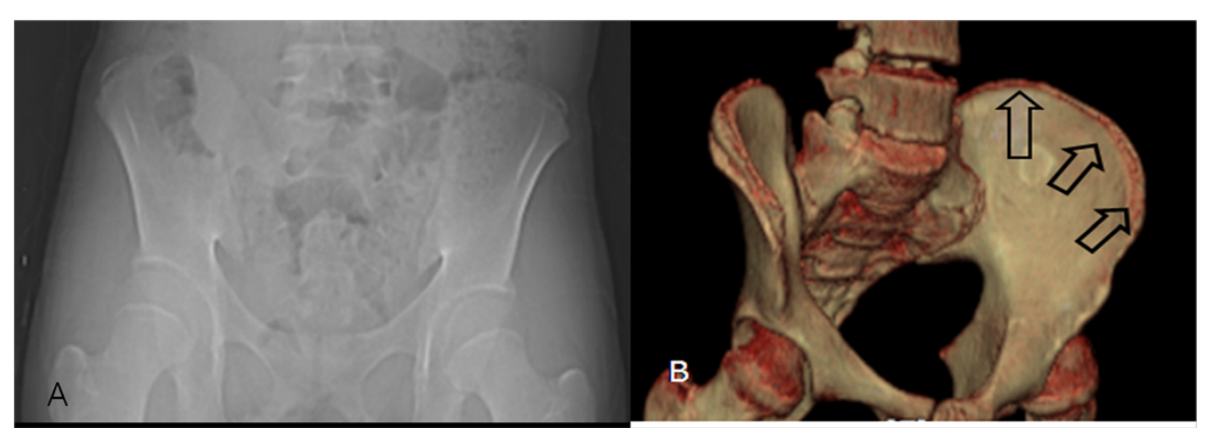

Figure 4 Inaccurate measurement of excursion. (A) Plain radiography performed on a 13-year-old girl; the apophysis excursion was visible covering approximately $40 \%$ of the iliac bone, and the patient was graded as Original Risser stage 2 and French Risser stage 1. (B) A 3D-CT image of the same patient; apophysis excursion was visible up to the posterior superior iliac spine and was graded as Original Risser stage 4 and French Risser stage 3. 


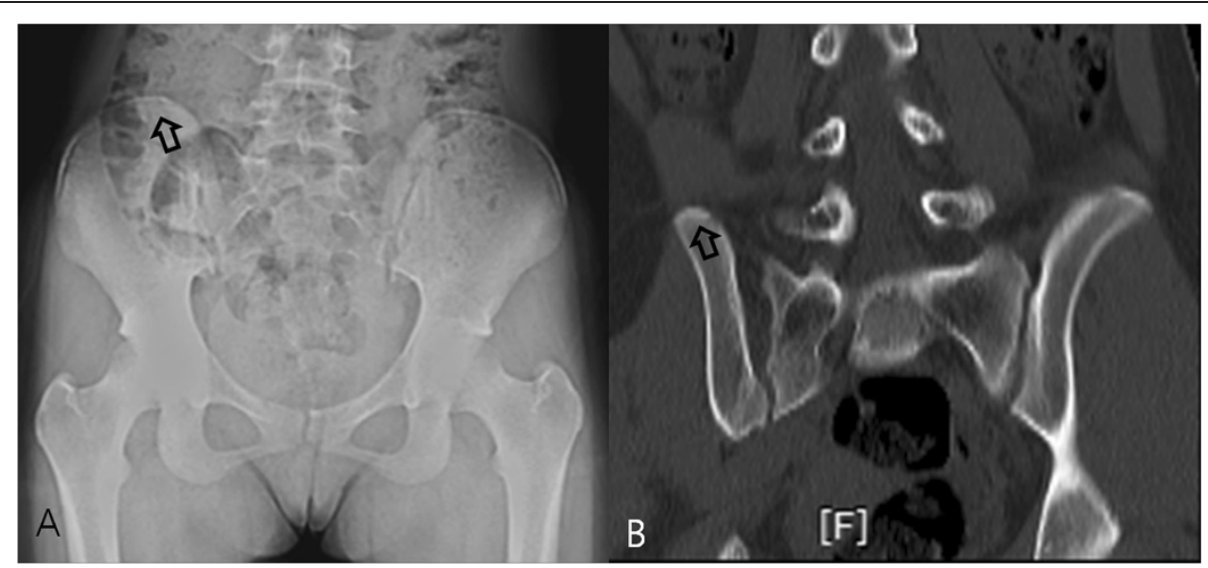

Figure 5 Micro-fusion. (A) Plain radiography of a 14-year-old girl; a radiolucent line was visible between the apophysis and the posteromedial iliac bone and was graded as Original Risser stage 4 and French Risser stage 3. (B) A coronal 3D reconstruction view; there was absence of any radiolucent line indicating definite fusion, and the staging by Original method was 5 and by French method was 4 . Accurate estimation of fusion of iliac apophysis or micro-fusion is difficult on plain radiography.

seems to proceed from pseudo-fusion to micro-fusion to complete fusion. FM, which divides fusion into two stages, had a $100 \%$ concordance rate in stage 5.

Most of the potential sources of errors can be eliminated. Gas shadows can be easily eliminated by adequate bowel preparation before X-ray acquisition. Obtaining high quality X-rays and observing them against bright light will minimize the failure to detect micro-calcifications [18]. As suggested by some authors, ultrasonography can be a useful tool and can complement X-rays in the detection of micro-calcifications and the actual extent of ossifications and fusions without exposing patients to the risks associated with radiation $[19,20]$. The addition of extra lateral or oblique views, and considering every available, view will reveal some of the missed excursions of the apophyseal ossification and the actual extent of fusion [4,5]. The modification of Risser's staging to minimize the divisions of iliac apophysis might lead to a decrease in miscalculation of the apophyseal excursions. Further, increased knowledge and awareness of the common variations among scoliosis patients will help surgeons identify anomalous ossification patterns such as skipped ossification and the appearance of the first posterior-medial ossification and will enable them to appropriately stage cases. Since fusion appears to start as micro-fusion or pseudo-fusion and then proceed to full fusion, fusion-related errors can be minimized by dividing the fusion stages into two categories, similar to $\mathrm{FM}$, and by merging the posteromedial ossification stage with the first fusion stage.

Accordingly, we propose the following modified version of Risser's staging:

- 0- No ossification.

- 1- Appearance of ossification anywhere, in less than one-third of the iliac wing.

- 2- Ossification of more than one-third and equal to or less than two-thirds of the iliac wing without evidence of any fusion.

- 3- Ossification beyond two-thirds of the iliac wing and appearance of fusion

- 4- Fusion of more than one-third of the iliac crest.

- 5- Complete fusion.

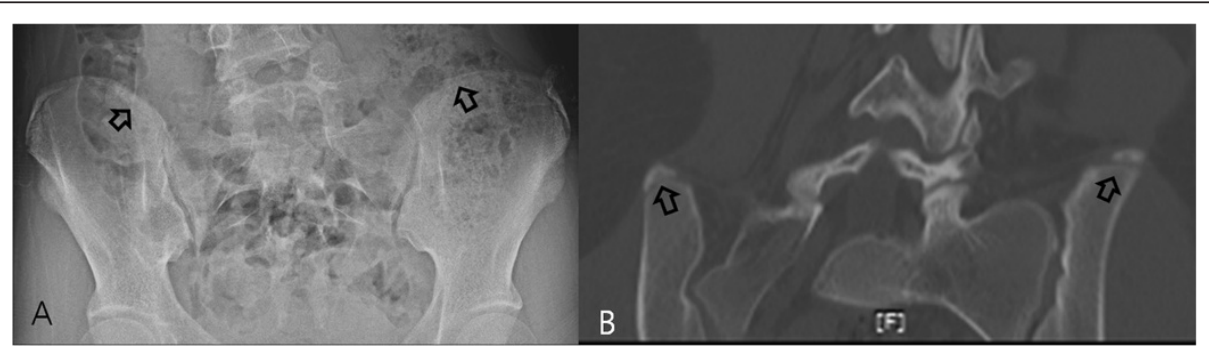

Figure 6 Pseudo-fusion. (A) Plain radiography of a 15-year-old girl; a sclerotic line was visible on the posterior iliac crest suggesting fusion of the iliac apophysis, and the patient was graded as Original Risser stage 5 and French Risser stage 4. (B) A coronal 3D reconstruction view; there was no definite fusion of the apophysis, and the patient was graded as Original Risser stage 4 and French Risser stage 3. Estimating the initiation of pseudo-fusion is difficult on plain radiography. 
Because 3D-CT results in exposure to radiation and has relatively high associated costs, PR was used to measure Risser's stage. However, a limitation of this study was that the new Risser's staging system was not objectively verified as a viable replacement for OM or FM. However, the inaccuracy of PR used in Risser's staging system was verified, using a different X-ray device. Moreover, the factors that caused measurement errors were analyzed, and a feasible solution was suggested. Considering that the verification of a new Risser's staging system requires collection of data and observational time, the presentation of a Risser's staging system using objective data would be meaningful.

We also acknowledge a limitation of this study was the uneven distribution of the enrolled patients according to Risser's stage, but we were careful not to overestimate or underestimate the results, and to indicate only the overall patterns. For this reason, we merely listed the main causes of error and did not perform detailed statistical analysis.

\section{Conclusions}

$P R$ for Risser's staging is reliable but is not a suitable tool for accurate measurement of the excursion of apophyseal ossification. $P R$ has limitations in determining the actual lengths of apophyseal ossification and fusion. We have proposed various guidelines to minimize errors and to increase the accuracy of Risser stage determination by $P R$, together with modifications to the Risser staging system.

\section{Ethical review committee statement}

The authors certify that this study involving human subjects is in accordance with the Helsinki declaration of 1975 as revised in 2000, and that the relevant institutional ethical committee approved the study.

\section{Competing interests}

The authors declare that they have no competing interests.

\section{Authors' contributions}

SWS carried out the arrangement of the enrolled patients and drafted the manuscript as an assistant to JHY. JHH participated in the design of the study and performed the statistical analysis as an assistant to JHY. SWS carried out the arrangement of the enrolled patients and drafted the manuscript as an assistant to JHY. AWB participated in the design of the study and helped with the preparation of the manuscript and its revision. JYH participated in the design of the study. JHY participated in the design of the study, the statistical analyses, and coordination, and drafted the manuscript as the primary author. $\mathrm{CHH}$ participated in the whole work of revision such as rechecking data, statistical analysis, and answering the queries of reviewers. All authors read and approved the final manuscript.

\section{Acknowledgements}

This study was supported by a grant from the Korea Healthcare Technology R\&D project, Ministry for Health, Welfare \& Family Affairs, Republic of Korea (A110416). The authors would like to thank the Ministry of Health, Welfare \& Family Affairs for allowing them to perform this research.

\section{Author details}

'Department of Orthopedics, Scoliosis Research Institute, Korea University Guro Hospital, Guro, Korea. ²Department of Orthopedics, Korea University Ansan Hospital, Guro, Korea. ${ }^{3}$ Division of Pediatric Orthopaedics, Orthopaedic Surgery, Yonsei University College of Medicine, Severance Children's Hospital, Seoul, Korea.

Received: 8 February 2014 Accepted: 9 October 2014

Published online: 19 November 2014

References

1. Risser JC: The lliac apophysis: an invaluable sign in the management of scoliosis. Clin Orthop 1958, 11:111-119.

2. Goldberg MS, Poitras B, Mayo NE, Labelle H, Bourassa R, Cloutier R: Observer variation in assessing spinal curvature and skeletal development in adolescent idiopathic scoliosis. Spine (Phila Pa 1976) 1988, 13:1371-1377.

3. Izumi Y: The accuracy of Risser staging. Spine (Phila Pa 1976) 1995, 20:1868-1871.

4. Kotwicki T: Risser sign: the value of the lateral spinal radiograph to assess the excursion of the iliac apophysis. Stud Health Technol Inform 2008, 140:44-47.

5. Kotwicki T: Improved accuracy in Risser sign grading with lateral spinal radiography. Eur Spine J 2008, 17:1676-1685.

6. Shuren N, Kasser JR, Emans JB, Rand F: Reevaluation of the use of the Risser sign in idiopathic scoliosis. Spine (Phila Pa 1976) 1992, 17:359-361.

7. Hettlich BF, Fosgate GT, Levine JM, Young BD, Kerwin SC, Walker M, Girffin J, Maierl J: Accuracy of conventional radiography and computed tomography in predicting implant position in relation to the vertebral canal in dogs. Vet Surg 2010, 39:680-687.

8. Learch TJ, Massie JB, Pathria MN, Ahlgren BA, Garfin SR: Assessment of pedicle screw placement utilizing conventional radiography and computed tomography: a proposed systematic approach to improve accuracy of interpretation. Spine (Phila Pa 1976) 2004, 29:767-773.

9. Ryan PJ, Evans PA, Gibson T, Fogelman I: Chronic low back pain: comparison of bone SPECT with radiography and CT. Radiology 1992, 182:849-854

10. Bitan FD, Veliskakis KP, Campbell BC: Differences in the Risser grading systems in the United States and France. Clin Orthop Relat Res 2005, 436:190-195.

11. Landis JR, Koch GG: The measurement of observer agreement for categorical data. Biometrics 1977, 33:159-174.

12. Sanders JO, Khoury JG, Kishan S, Browne RH, Mooney JF 3rd, Arnold KD, McConnell SJ, Bauman JA, Finegold DN: Predicting scoliosis progression from skeletal maturity: a simplified classification during adolescence. J Bone Joint Surg Am 2008, 90:540-553.

13. Little DG, Sussman MD: The Risser sign: a critical analysis. J Pediatr Orthop 1994, 14:569-575.

14. Biondi J, Weiner DS, Bethem D, Reed JF 3rd: Correlation of Risser sign and bone age determination in adolescent idiopathic scoliosis. $J$ Pediatr Orthop 1985, 5:697-701.

15. Reem J, Carney J, Stanley M, Cassidy J: Risser sign inter-rater and intra-rater agreement: is the Risser sign reliable? Skeletal Radiol 2009, 38:371-375

16. Dhar S, Dangerfield PH, Dorgan JC, Klenerman L: Correlation between bone age and Risser's sign in adolescent idiopathic scoliosis. Spine (Phila Pa 1976) 1993, 18:14-19.

17. Hammond KE, Dierckman BD, Burnworth L, Meehan PL, Oswald TS: Inter-observer and intra-observer reliability of the Risser sign in a metropolitan scoliosis screening program. J Pediatr Orthop 2011, 31:e80-e84.

18. Zaoussis $A L$, James Jl: The iliac apophysis and the evolution of curves in scoliosis. J Bone Joint Surg Br 1958, 40-B:442-453.

19. Thaler M, Kaufmann G, Steingruber I, Mayr E, Liebensteiner M, Bach C: Radiographic versus ultrasound evaluation of the Risser Grade in adolescent idiopathic scoliosis: a prospective study of 46 patients. Eur Spine J 2008, 17:1251-1255.

20. Torlak G, Kiter E, Oto M, Akman A: Ultrasonographic evaluation of the Risser sign. Spine (Phila Pa 1976) 2012, 37:316-320.

doi:10.1186/s13018-014-0101-8

Cite this article as: Yang et al:: Evaluation of accuracy of plain radiography in determining the Risser stage and identification of common sources of errors. Journal of Orthopaedic Surgery and Research 2014 9:101. 